\title{
VIRAL DISEASES OF ZOONOTIC IMPORTANCE OF DOIMESTIC RUMINANTS
}

Reda M.S. Korany and Sherein S. Abdelgayed

Department of Pathology, Faculty of Veterinary Medicine, Cairo University, Giza, 12211, Egypt

*Corresponding author: sherein.abdelgayed@vet.cu.edu.eg

\section{INTRODUCTION}

Rudolf Virchow, one of the $19^{\text {th }}$-century German leading scientists in medicine and pathology, noted a relationship between human and animal diseases and introduced the term "zoonosis" in 1880 (Walter and MikeScott 2017). Later, the World Health Organization (WHO) in 1959 defined zoonoses as the diseases and infections that are naturally transmitted between vertebrate animals and man (Al-Tayib 2019). Zoonotic agents can cause different kinds of diseases, causing major public health issues worldwide (Ermias et al. 2017).

Zoonoses are a group of infectious diseases that are transmitted from animals to humans (zooanthroponoses) or from humans to animals (anthropozoonoses). Ruminant animals such as cattle, buffaloes, sheep and goats are considered as an important source of infections for humans due to their close contact and also due to their importance to the human society (Ganter 2015). Zoonotic diseases can adversely affect public health through reduction in livestock productivity. Zoonotic diseases can be transmitted naturally from animals to humans with or without arthropod intermediates (Al-Salihi 2018). Such diseases include multiple infections like viral, bacterial, fungal, protozoal and parasitic diseases (Al-Tayib 2019). Viral zoonotic pathogens are important in emerging and reemerging virus diseases (McDaniel et al. 2014), and are found in all continents except Antarctica. Some of them are found all over the world, while others in limited ecological areas. There are numerous viral zoonotic species which are considered as important livestock pathogens (Reed 2018).

Viruses are a group of pathogens that are unable to reproduce outside the host cell and always need the host DNA to complete their life cycle, so they are considered as obligate intracellular organisms. A virus consists of DNA or RNA segment and is encircled by a protein coat with or without viral membrane. Virulence factors are essential for viral replication and also for protection against the host immune system. Viruses are excreted by clinically or sub-clinically infected hosts into the environment, where they remain in a dormant state. Survival of viruses in the environment is an important factor concerning their virulence and pathogenicity. Also, their ability to induce genetic changes enables them to have a wide range of hosts. Transmission of viral zoonotic diseases from animals to humans occurs in many ways, such as direct contact (e.g. milker's nodule), airborne transmission (respiratory viruses e.g.
$\mathrm{H}_{1} \mathrm{~N}_{1}$ ) and mosquito-borne like Rift Valley fever RVF) virus (Garvey 2018).

\section{Rift Valley Fever}

Rift Valley fever (RVF) is an acute febrile zoonotic infection that affects sheep, goats, buffaloes, cattle and humans. Its causative agent is an RNA virus, Phlebovirus (family Bunyaviridae). The first record of this disease was noted in 1930, when animal and human affections appeared in a farm in Naivasha Lake, in the Rift Valley after heavy rainfall due to increased number of mosquitos. Many outbreaks of Rift Valley fever were recorded in 19oos. Five large outbreaks of the disease were recorded in Egypt during the last 40 years; in 19771978, 1993-1994, 1997, 2000 and 2003, with the largest epizootic was in 1977-1978. The most severe outbreaks were in 1975 in South Africa and during 1977 in Egypt (Helmy et al. 2017). Currently, the disease has economic importance due to the cost of preventive measures, monitoring for introducing the disease to disease-free areas, and restrictions on the import and export of animals (Lichoti et al. 2014).

\section{Epidemiology}

\section{Transmission and environmental risk factors}

The Rift Valley Fever Virus (RVFV) is a mosquito-borne virus. Once cattle and sheep get infected, they become highly viraemic, allowing efficient viral transmission. Trans-ovarian transmission of Rift Valley fever virus is another feature. Infected eggs of mosquitos can survive for many years in the soil. Under suitable environmental conditions, such as heavy rainfall that creates stagnant water, mosquito eggs will hatch and result in the production of newly infected mosquitos. In the East and South Africa, the main vector for RVF is Aedes mosquito. RVFV can withstand environmental temperatures up to $25-30^{\circ} \mathrm{C}$ for about 80 minutes. Humans can get infected by contact with infected animals and animal products in the abattoir, and the areas of food preparation in endemic areas, but less frequently as a result of a mosquito bite. Human to human transmission has not been recorded (Nakoune et al. 2016).

\section{Geographic distribution}

RVFV affects ruminants in Africa, but it can spread to areas other than Africa and can be a worldwide threat for 


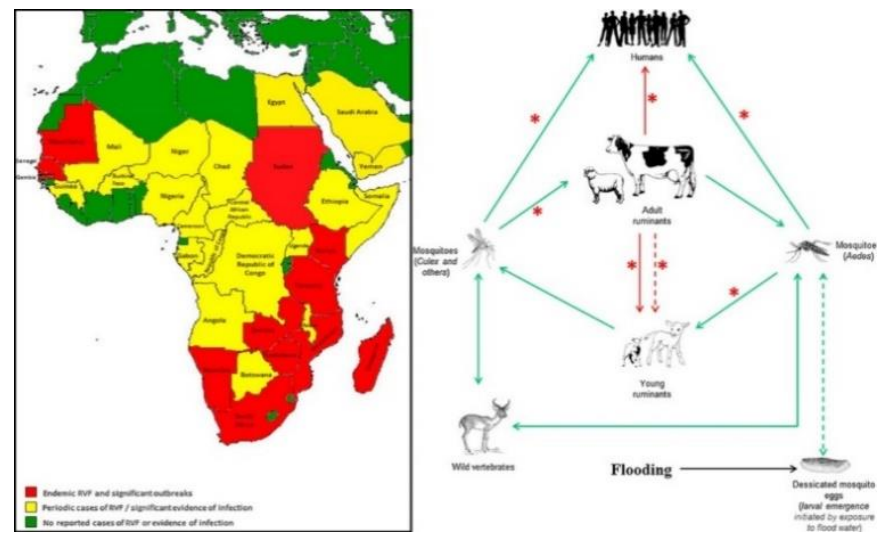

Fig. 1: Geographic distribution of RFV (http://www.cdc.gov/vhf/rvf/distribution-map.html) (left). Diagram of the transmission cycle of RVFV (https://www.pasteur.fr/en/research/virology/unitsgroups/arboviruses-and-insect-vectors/research) (right).

humans and animals (Fig. 1). The spread of the virus to the Arabian Peninsula in 2000 and Madagascar and Comoros in 2007-2008 shows the possibility for its spreading to many areas. RVF has been reported in four ecologic systems: irrigated areas, Dambo areas, semi-arid areas and temperate and mountainous areas (Kasye et al. 2016).

\section{Host range and susceptibility}

RVF virus was reported in antelopes, buffaloes, cattle, goats, camels, monkeys, rodents and sheep, in addition to human. Mortality and morbidity have been recorded in sheep, cattle and humans. Young animals and humans are highly susceptible to RVF infection. However, reptiles and Amphibians are resistant to this virus (Shayif et al. 2018).

\section{Morbidity and mortality}

Young animals and humans are highly susceptible to RVFV with a mortality of $70-100 \%$. Adult animals and humans are moderately susceptible with a mortality rate of $<10 \%$; case fatality rate in humans is $<1 \%$. Equines, dogs, pigs and cats are considered to be resistant (Métrasa et al. 2020).

\section{Source of infection}

Tissues of viremic animal, blood, aborted fetus and fomites are considered to be the main sources of RVF virus infection (Petrova et al. 2020).

\section{Pathogenesis}

RVFV replicates in mosquitos, invertebrate animals and humans. After an insect bite, RVFV is transported by lymphatics from inoculation site to regional lymph node, where it replicates and is conveyed to the circulation with initiation of viremia and systemic response. Liver, brain and spleen are the main target sites for viral replication. Virus is resistant to alkaline environment and is inactivated by disinfectants such as sodium hypochlorite, acetic acid and calcium hypochlorite. It can be for eight years when stored below $0^{\circ} \mathrm{C}$ (Javelle et al. 2020).

\section{Clinical Signs in Cattle}

Incubation period of RVFV is about $24-72$ hours in adult cattle. Calves become feverish $\left(40-41^{\circ} \mathrm{C}\right)$, off-feed, weak, depressed, diarrheic with jaundice. Adults often show unapparent infection; although fever lasting for 24 to 96 hours, nasal discharge, lacrimation, dull coat, excessive salivation, weakness, anorexia, low milk production, bloody diarrhea, and abortion in pregnant cows are common signs (Kasye et al. 2016).

\section{Clinical Signs in Small Ruminants}

Among small ruminants, the incubation period of RVF virus is 12 to 72 hours in newborn lambs and 24 to 72 hours in adult sheep and goats. Newborn lambs of $<2$ weeks age show biphasic fever (about $40-41^{\circ} \mathrm{C}$ ), weakness, anorexia, rapid respiration and abdominal pain, with death occurs within 24-36 hours. Lambs aged more than 2 weeks, as well as adult sheep and goats, show fever for 24 to 96 hours, depression, increased respiratory rate, weakness, anorexia, bloody diarrhea, vomiting, mucopurulent nasal discharge, jaundice and abortion in almost $100 \%$ cases (Kasye et al. 2016).

\section{Clinical Signs in Humans}

Incubation period of RVF virus is 3 to 6 days in humans. RVF in humans is mostly asymptomatic. Sometimes they develop a self-limiting fever with flu-like signs generally characterized by fever, headache, muscular pain, nausea and photophobia. Recovery may occur within 4 to 7 days. Some patients may develop a hemorrhagic fever and jaundice with case fatality about $0.5-2.0 \%$. Neurological disorders and blindness are rare complications (Javelle et al. 2020).

\section{Pathologic Lesions}

Primary lesion in RVF infection is liver necrosis. In aborted fetus and neonates, the liver is soft, friable, enlarged and dark in color. Enlarged and edematous peripheral and visceral lymph nodes, hemorrhages and edema in gall bladder, hemorrhagic enteritis, cutaneous hemorrhages, blood-stained fluid accumulated in body cavities with subcutaneous and serosal hemorrhages are also observed (Kasye et al. 2016).

\section{Clinical Diagnosis}

Affected animals show fever, depression, mucopurulent nasal discharge, anorexia, vomiting, jaundice, weakness and bloody diarrhea. Clinical pathology reveals severe leucopenia, high serum levels of glutamic dehydrogenase (GLDH) due to liver damage and also thrombocytopenia (Petrova et al. 2020).

\section{Laboratory Diagnosis}

Laboratory diagnosis of RVF depends on serology by detecting the antibodies, either IgM or by detecting the 
rising titer of IgG. In encephalitis, cerebrospinal fluid can be evaluated for IgM. RVFV is isolated from blood in the acute phase. PCR is also used either in serum or tissue (Petrova et al. 2020).

\section{Treatment}

For mild and moderate cases of RVF, analgesics and fluid therapy can be administered; for severe cases as in encephalitis and hemorrhage, critical care like ventilation and blood transfusion is necessary (Kasye et al. 2016).

\section{Prevention and Control}

Two vaccines for RVF are available and are commonly used in endemic areas; one is a live attenuated Smith burn vaccine and the other is a formalin-inactivated vaccine. Mosquitoes are the most important vectors for RVF virus. Only female mosquitos feed on blood and lay their eggs near water. The eggs hatch into larvae, which are transformed into pupae. The larvae and the pupae need water to survive. Adult female mosquitoes lay eggs in specific areas (near water sources), so they can be easily managed. Mosquito larvae remain in the same areas, so control of these stages depends on continued management of these areas. Adult mosquitoes can be controlled by the use of insecticides. But it is difficult and expensive way. Also, sterilization of male mosquitoes by radiation may be effective. Ban on the movement of animals from the suspected areas and confinement of animals in covered areas are advised as a preventive measure. Attempts may also be made to prevent the lambing and calving during the rainy season. Use of protective clothes and insect repellents and prevention of outdoor activities during mosquito biting season are essential protective measures. Quarantine practice on entering or leaving the suspected areas is also recommended (Boshra et al. 2011).

\section{Crimean-Congo Hemorrhagic Fever}

Crimean-Congo hemorrhagic fever (CCHF) is a fatal tickborne disease; it is a widespread viral infection. The CCHF virus is maintained in ticks via horizontal and vertical transmission and transmitted to domestic animals, which can convey the disease to humans. In 1944, CCHF disease was first recorded in Crimea. Then in 1969, it was reported from the Congo region, so the disease was named as "Crimean-Congo hemorrhagic fever". The CCHF virus belongs to genus Nairovirus and family Bunyaviridae. It is a single-stranded RNA virus and exists in seven genotypes, namely Africa-1, Africa-2, Africa-3, Europe-1, Europe-2, Asia1 and Asia-2 (Mostafavia et al. 2017).

Epidemiology

\section{Transmission and environmental risk factors}

Person to person transmission of CCHF virus occurs via direct contact with body fluids of infected persons and also via contact with the animal blood and animal products. Nosocomial infection among health care workers, especially during hemorrhagic phase, is common. Hyalomma tick is the vector of the virus. Adult virus obtains its nutrition from cattle, sheep and other large mammals. Migration of the infected livestock from infected area to free area is another factor. Trans-ovarian transmission is essential to maintain a large number of infected ticks. Spread of the virus between ticks and animals is high during summer and spring seasons, when ticks larvae and nymphs develop into adults. Biting results in the transmission of virus from the tick to the host. Human is a dead-end host for the virus. Droplet infection is also considered one of the methods of disease transmission (Aslam et al. 2016).

\section{Geographic distribution}

The CCHF virus is characterized by widespread distribution (Fig. 2) and it must be considered as global health threat. The disease is distributed in different areas, including Africa, Asia, central and southern Europe, Eastern Europe, throughout the Mediterranean, north-western China, the Middle East and the Indian subcontinent. Since 2002, the virus has also been found in many countries of the Balkans. From 2000-2008, the disease re-emerged in Albania, Bulgaria, Turkey and Kosovo. It has also been recorded in Greece (Zavitsanou et al. 2009).

\section{Host range and susceptibility}

Infected humans can spread the infection through close contacts. Domestic animals including cattle, sheep, goats, horses, ostriches, pigs, donkeys, camels, mice and domestic dogs are considered as the main hosts for the CCHF virus and can transmit it to humans during viraemic phase. Slaughtering and animal handling can facilitate the transmission of the infection. Also, healthcare workers are at risk. Rural area is a risky place for exposure to ticks. Risk is also high among people in urban areas such as animal trading markets (Munibullah et al. 2018).

\section{Morbidity and mortality}

The CCHF is highly pathogenic, with a high case fatality rate $(10-40 \%)$. The prevalence of $\mathrm{CCHF}$ in people with history of tick bite can reach $20 \%$ in endemic areas (Shayan et al. 2015).

\section{Source of infection}

The CCHF disease virus is mainly in hemorrhages from mouth, gums, nose, rectum, urinary bladder, urethra and vagina (Parmar et al. 2017).

\section{Pathogenesis}

Hepatocytes and Kupffer cells are major target cells for CCHF virus. Hepatocellular necrosis leads to elevated liver enzymes. Also, elevated myeloperoxidase expression 


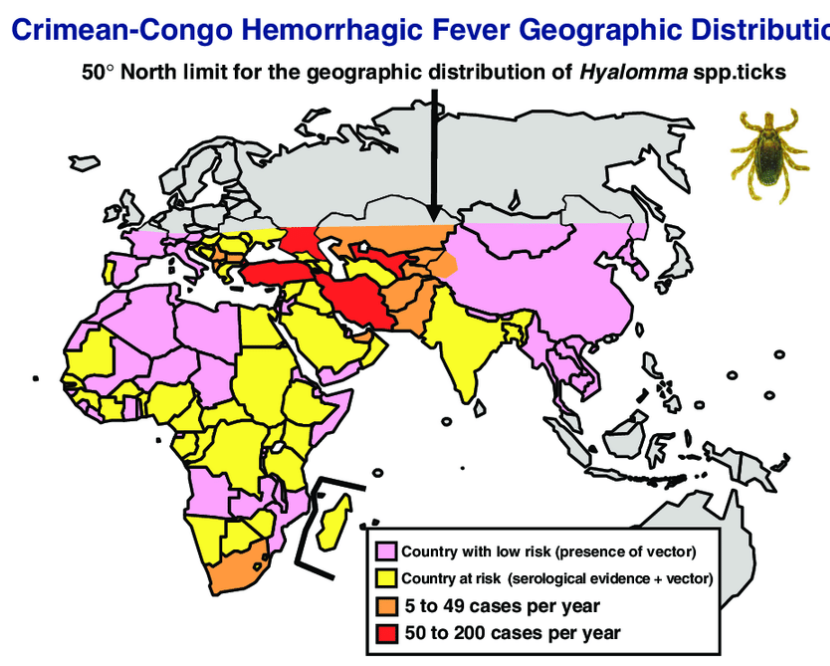

Fig. 2: Geographic distribution of $\mathrm{CCHF}$ (https://www.researchgate.net/publication/226967229).

in leukocytes leads to increased leukocytic lysis, leading to leukopenia. Endothelial damage activates the coagulation cascade, resulting in decreased platelets count. Endothelial damage also leads to hemostatic failure and skin rashes. Activation of coagulation may have a role in the development of disseminated intravascular coagulation. Infection by virus or damage by secreted cytokines leads to bleeding (Aslam et al. 2016).

\section{Clinical Signs in Cattle}

Incubation period of CCHF virus is 3-7 days. There are four stages of the infection, incubation period (asymptomatic phase), pre-hemorrhagic, hemorrhagic and convalescent (symptomatic phase). Pre-hemorrhagic phase extends from 4-5 days, and is characterized by fever, abdominal pain, headache, hypotension, myalgia, and flushed face. As disease progresses, petechial and ecchymotic hemorrhages, epistaxis, gum bleeding and hematemesis start. Nausea, diarrhea, neuropsychiatric and cardiovascular dysfunction may appear. The convalescent phase starts in survivors about 10-20 days after the disease. Recovery can take around one year period in survivor animals (Zavitsanou et al. 2009).

\section{Clinical Signs in Humans}

In human, incubation period of CCHF virus depends on the mode of infection. After insect bite, incubation period is about 1-3 days, with maximum of 9 days. Following contact with infected blood or tissue, the incubation period is usually about 5-6 days, with maximum of 13 days. The pre-hemorrhagic period starts with abrupt fever, dizziness, abdominal and back trouble and harsh head pain. Other signs include vomiting, nausea, diarrhea and cardiovascular and neuropsychiatric alterations. This phase ends after 3 days. Then the characteristics GIT blood loss, respiratory and urinary tract bleeding, blood loss also occurs from skin, ranging from petechiae to ecchymosis. Death occurs due to multiple organ failures (Munibullah et al. 2018).

\section{Pathologic Lesions}

Hepatomegaly and splenomegaly are characteristic necropsy findings in $\mathrm{CCHF}$ cases. Moreover, cerebral hemorrhage, lung edema and pleural effusion are also seen (Shayan et al. 2015).

\section{Clinical Diagnosis}

Besides clinical symptoms, history of insect bite and exposure to tissue or blood of infected animals and humans and also travel to the endemic region are important considerations for diagnosis of CCHF disease. Leucopenia, thrombocytopenia, and increased aspartate aminotransferase, alanine aminotransferase, creatinine phosphokinase and lactate dehydrogenase activity values are helpful. Prolonged prothrombin time, activated partial thromboplastin time, decreased fibrinogen and increased fibrin degradation products are also helpful(Munibullah et al. 2018).

\section{Laboratory Diagnosis}

Enzyme-linked immunosorbent assay (ELISA), antigen detection, quantitative polymerase chain reaction (qPCR), serum neutralization and virus isolation are useful tools for laboratory diagnosis of CCHF infection. After first day of viremia, weak IgM is detectable, followed by IgG. Immunohistochemistry is also used to detect the virus (Aslam et al. 2016).

\section{Treatment}

There is no drug of choice against CCHF virus infection. However, ribavirin is the only antiviral agent that can be taken orally and intravenously. Supportive therapy like interferons is also used. Immunoglobulin, venin is specific drug for CCHF disease (Zavitsanou et al. 2009).

\section{Prevention and Control}

There is no available vaccine for $\mathrm{CCHF}$ virus infection. Chances of tick exposure may be reduced by avoiding areas and seasons of high prevalence. High-risk occupation people such as butchers, shepherds and veterinarians should avoid ticks and suspected animal blood and tissue exposure. The use of gloves and protective clothes is also an effective measure. The medical workers should deal with care with suspected patients. Proper processing of milk and food should be done. Treatment of the livestock with acaricide is helpful in reducing the populations of insects. Insect repellents use on skin is also needed. Quarantine measures should be performed when importing animals. Control measures should be taken during slaughtering, butchering and culling. The equipment should be washed after slaughtering. Avoid close contact with the infected persons (Munibullah et al. 2018). 


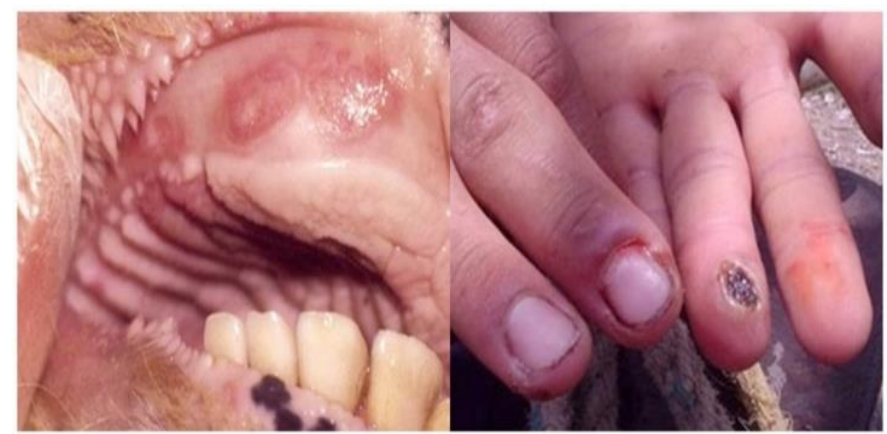

Fig. 3: Bovine Papular Stomatitis in cattle (left), available at https://veteriankey.com/alimentary-disorders/, and in human (right), available

at http://coloradodisasterhelp.colostate.edu/prefair/disease/dz/Bo vine\%2oPapular\%2oStomatitis.html

\section{Bovine Papular Stomatitis}

Bovine Papular Stomatitis (BPS) is known as a granular or proliferative stomatitis. It is an infectious disease, affecting cattle of all ages and has been previously reported in many countries. It is caused by Bovine Pustular Stomatitis Virus (BPSV), belonging to Genus Parapoxvirus and Family Poxviridae. Parapox is an enveloped, oval and double-stranded DNA virus. BPSV has the potential to infect the human; therefore, it is considered as a zoonotic disease (Pal 2020).

\section{Epidemiology}

\section{Transmission and environmental risk factors}

Virus transmission is by direct contact between infected and susceptible animals. Zoonotic Parapoxvirus infection can cause the disease in milkmen (Fig. 3) and farmers (milker's nodules). Humans get the infection from direct contact with the infected cattle. An incidental bite during examination of diseased cattle can also result in human infection. Virus may also enter the body through a pre-existing skin lesion. The BPS virus mainly causes lesions in immunocompromised cattle or calves with a concurrent disease (Underwood et al. 2015).

\section{Geographic distribution}

Bovine Pustular Stomatitis is a widespread disease prevalent in many countries, such as Australia, Canada, Brazil, Europe, Nigeria, Kenya, South Korea and USA. It is responsible for great economic losses in dairy farms and also for the problems related to public health (Gelberg 2017).

\section{Host range and susceptibility}

The BPS virus can affect cattle of all ages; although its incidence is higher in cattle less than two years of age. Concerning human beings, BPS is considered as an occupational zoonotic infection (Peek et al. 2018).

\section{Morbidity and mortality}

BPS shows a high morbidity rate in severe outbreaks. It has low mortality rate; although, it can cause huge economic losses to the dairy industry (Underwood et al. 2015).

\section{Clinical Signs in Cattle}

The infected cattle shows papular and pustular rashes on the skin of muzzle, lips, nose, and buccal mucosa, with profuse salivation, fever, and diarrhea. Occasionally, teats of milking cows are also affected and show painful reddish papule, ulcer and scabby proliferative lesion (Senturk et al. 2016).

\section{Clinical Signs in Humans}

After milking of affected cows, milk-men show severe lesions on the fingers, hands, arms and face, besides axillary lymphadenopathy. The lesions of BPS are painful papules and pustules that can progress to ulcers and scabby lesions (de Sant'Ana et al. 2012).

\section{Pathologic Lesions}

Necropsy findings usually correspond to the lesions in live animals and are characterized by presence of papules and/or pustules on muzzle, lips, nose, and buccal mucosa. Teats of milking cows also show papules, ulcers and scabby lesions (de Sant'Ana et al. 2012).

\section{Clinical Diagnosis}

Clinical signs are not characteristic to give an accurate diagnosis of BPS (Underwood et al. 2015).

\section{Laboratory Diagnosis}

Laboratory diagnosis of BPS infectionincludes isolation of the virus in tissue culture, serology, polymerase chain reaction (PCR) and electron microscopy. The disease should be differentiated from the Pseudocowpox (Gelberg 2017).

\section{Treatment}

The disease is self-limiting so, no treatment is required. However, antibacterial and antibiotic creams may be applied to the skin lesions to prevent secondary bacterial infection (Peek et al. 2018).

\section{Prevention and Control}

Control measures, such as care in handling of infected cattle, wearing the gloves during examination of infected animals, proper attention to the skin lesions, thorough washing of hands with antiseptic solutions, and quarantine of the newly purchased animals help to control BPS infection. Awareness of such zoonotic diseases among the livestock handlers should be created. Moreover, early recognition of infection is necessary to prevent its further spread (Underwood et al. 2015). 


\section{Foot and Mouth Disease}

Foot and Mouth Disease (FMD), hoof and mouth disease or Aphthae epizooticae, is an infectious or even fatal (contagious) disease of cloven-hoofed animal (wild and domestic bovid). This disease is a severe plague for animal farms, as it is highly infectious. The virus responsible for the disease is picornavirus, a member of the genus Aphthovirus. It is a single-stranded RNA virus having seven serotypes, including $\mathrm{O}, \mathrm{A}, \mathrm{C}, \mathrm{SAT} 1, \mathrm{SAT}$, $\mathrm{SAT}_{3}$ and Asiaı (Farsang et al. 2013).

\section{Epidemiology}

\section{Transmission and environmental risk factors}

FMD is a highly transmissible viral infection, as a limited number of virus particles can initiate the infection. Transmission is by direct contact with contaminated animal products, materials, contaminated clothes and footwear of workers or contaminated equipment. Aerosol spread has also been recorded. Humans can acquire the infection by contact with infected animals or via ingestion of raw milk and dairy products from infected cows (Fig. 3). However, humans rarely acquire FMD infection. The virus enters the body via injured skin and mucous membrane. No person-to-person transmission has been recorded. The atmospheric conditions can either provide a barrier to virus dissemination or can promote its transmission. The virus persists for days or even weeks in organic matters under moist and cold conditions. Also, it can survive in lymph nodes, frozen bone marrow, and cheese during its processing. It can be inactivated by citric acid (0.2\%), acetic acid ( $2 \%)$, sodium chloride $(2 \%)$, sodium carbonate $(4 \%)$, sodium hypochlorite $(3 \%)$ and sodium hydroxide (2\%). The virus in animal products can be inactivated by heating at $70^{\circ} \mathrm{C}$ for at least 30 minutes (Elmeligy 2017).

\section{Geographic distribution}

FMD is endemic in the Middle East, Africa, Asia and South America. New Zealand, North America, Iceland, most of Europe, Australia and Greenland are free of the disease. The last outbreak in United States occurred in 1929 (Osmani et al. 2019).

\section{Host range and susceptibility}

FMD has a wide host range and a rapid spread pattern. Bison, buffalo, alpaca, antelope, chamois, coypu, camel, cattle, deer, elephant, moose, pig, llama, reindeer, elk, giraffe, goat, sheep, and yak are among suspected animals. Laboratory workers, dairy farmers, veterinarians and cattle owners are also at risk of getting the infection (Weaver et al 2013).

\section{Morbidity and mortality}

In cattle, morbidity is about $100 \%$ but mortality is only about $2 \%$. However, mortality in calves can reach $20 \%$.
The death in calves may be due to myocarditis. Carrier state in cattle varies from 15 to 50\% (Elmeligy 2017).

\section{Source of infection}

FMD virus is found in secretions and excretions of infected animal. The virus persist in milk and semen for 4 days

before appearance of clinical symptoms. Also, contaminated animal products, agricultural tools, vehicles and people can contribute to the mechanical transmission of the virus (Rweyemamu et al. 2008).

\section{Pathogenesis}

The FMD virus is taken into the host cell. The cell produces multiple virus copies and then bursts to release the new virus in the blood (Arzt et al. 2011).

\section{Clinical Signs in Cattle}

Incubation period of FMD is about 2-14 days. The infection is manifested by fever that declines after 2-3 days, with the appearance of vesicles on feet, in and around mouth, and on mammary glands. Vesicles may also appear on vulva, prepuce or pressure points on legs. Vesicles rupture, leaving erosions. Depression, anorexia, lameness, excessive salivation and difficult to move are also frequent signs. Adult animals may suffer from weight loss. In cows, milk production can be decreased significantly; the disease may lead to myocarditis and death, especially in calves. Some animals remain asymptomatic but act as carriers for the infection and can transmit the disease to other animals (Arzt et al. 2011).

\section{Clinical Signs in Humans}

Incubation period FMD virus in humans is about 2-6 days. Signs of the disease include fever, vomiting, malaise, red ulcers on the oral mucosa, and vesicular lesions on the skin. In England, FMD killed two children in 1884 due to ingestion of infected milk (Bauer 1997).

\section{Pathologic Lesions}

Necropsy findings of FMD are characterized by presence of vesicles and/or erosions in the mouth, on the tongue, feet and mammary glands. Vesicles may also appear on the external genital organs. Erosions also may be found in rumen with necrotic myocarditis in some cases (Arzt et al. 2011).

\section{Clinical Diagnosis}

FMD infection in animals should be differentiated from infectious bovine rhinotracheitis, malignant catarrhal fever, contagious ecthyma, bovine viral diarrhea, bluetongue, bovine papular stomatitis, vesicular exanthema, vesicular stomatitis and mucosal disease. 
Also, FMD should be differentiated from infections with many viruses of the Coxsackie A group (hand and mouth disease) (Elmeligy 2017).

\section{Laboratory Diagnosis}

The ideal samples for laboratory diagnosis of FMD are vesicular fluid and epithelium, deep swabs or scrapings of erosions, nasal swab and acute and convalescent serum sample. The diagnosis of FMD depends on the isolation of the virus from clinical material. ELISA, complement fixation test (CFT) and serum neutralization test are used for diagnosis of FMD. Detection of antibodies by agar-gel immunodiffusion, antibody detection ELISA and virus neutralization, new technology of nucleic acid detection can also be used with good results (Martinez-Salas et al. 2008).

\section{Treatment}

There is no specific treatment for FMD and recovery usually occurs within 15 days. In humans, the disease is often mild and self-limiting, and recovery usually occurs within one week, antibiotic or antiseptic ointment should be applied to the skin lesion to prevent the secondary bacterial infection. Drugs that enhance immunity and systemic antibiotic course may be helpful (Elmeligy 2017).

\section{Prevention and Control}

FMD should not be considered a public health threat. Animals with natural or vaccine-mediated immunity can act as a carrier if exposed to the infection; such animals will remain asymptomatic. Certain measures such as quarantine and destruction of infected livestock and use of gloves during examination of the diseased cattle are helpful. Attempts should be made to avoid direct contact with diseased animal, visiting the farms in affected areas and consumption of raw milk and dairy products. Moreover, disinfection of clothes, materials, vehicles premises and equipment, with regular immunization of animals are also advised. However, one of the major difficulties in vaccination is that FMD virus continually evolves and mutates; there is no cross protection between different virus serotypes. Vaccination provides only temporary immunity. Proper disposal of carcasses by incineration or deep burial also helps. Immediate report of an outbreak of FMD in non-endemic areas should be made so that strategies for its control can be undertaken in time (Farsang et al. 2013).

\section{Contagious Ecthyma-ORF}

The disease contagious ecthyma-ORF is also named as contagious pustular dermatitis, scabby mouth, sore mouth, infectious labial dermatitis, ecthyma contagiosum, and thistle disease. It is a highly contagious and zoonotic disease which affects small ruminants (sheep and goats) and also other domestic and wild ruminants. It is one of the notifiable viral diseases. The causative agent of contagious ecthyma is a doublestranded DNA virus, belonging to genus Parapoxvirus and family Poxviridae. Other members of this genus are pseudopoxvirus, bovine viral stomatitis virus and Parapoxvirus of red deer in New Zealand. Four strains of the virus have been recognized, OVIA82 and OVSAoo in America, D1701 in Germany and NZ2 in New Zealand (Tedla et al. 2018).

\section{Epidemiology}

\section{Transmission and environmental risk factors}

Human infection of contagious ecthyma is usually occupational. The virus is conveyed from animals to humans by direct contact or by exposure to fomites. Virus infections in humans occur when injured skin comes in contact with infected animals or contaminated equipment. The virus is highly resistant to adverse environmental conditions and can persist for many years. Human to human transmission has not been recorded. Transmission through aerosol route, ear tagging and contaminated gavage is also possible (Teshale and Alemayehu 2018).

\section{Geographic distribution}

ORF is a worldwide infection and it is common in late summer, fall and winter. Its seasonality is related to the increased stress from lambing or cold weather. ORF was firstly reported in 1920 from South West Africa. After that, it has been recorded in all parts of the world that rear sheep and goats. Outbreaks of infection have also been recorded from Norway, Brazil, China, Ethiopia, Indonesia, Spain and Iraq (Kumar et al. 2015).

\section{Host range and susceptibility}

ORF is an infection of sheep, goats and other domestic and wild ruminants. Incidences of human infection were reported in many countries, including the United Kingdom. Risk factors include; age, increased orphaned lambs, increased stocking density, immunosuppression, prolonged parturition and thorny plant (Taghipour et al 2015).

\section{Morbidity and mortality}

With first exposure of ORF, morbidity can reach $70 \%$, while mortality rates are usually lower than $1 \%$, although higher mortalities (90\%) have been recorded in lambs with secondary bacterial infection. The internal organs affection is rare; however, some sheep and goats, which are severely infected with ORF, are found dead. The disease is severe in young animals, as they can die of starvation (Joseph et al. 2015).

\section{Source of infection}

Skin lesions and contaminated fomites, such as food and water and contaminated equipment are considered as the main sources of transmission of the infection (Karki et al. 2019). 


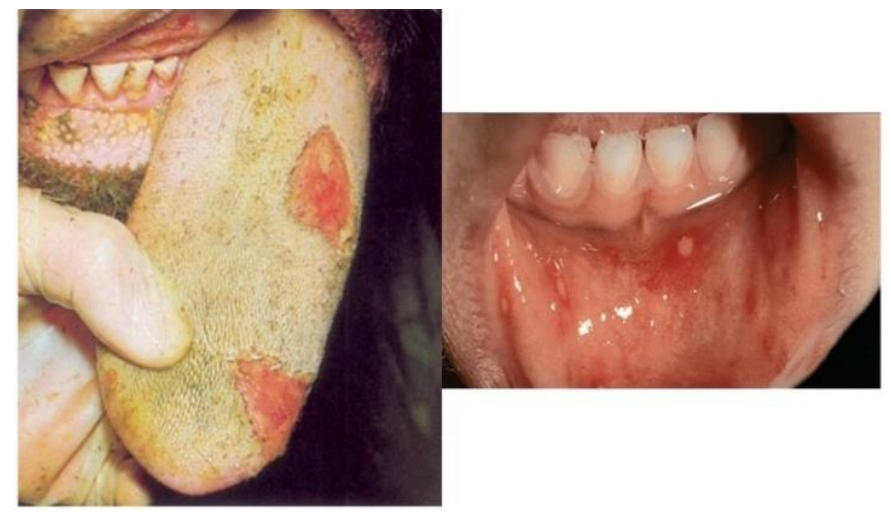

Fig. 4: FMD in cattle tongue (left), available at https://www.business.qld.gov.au/industries/service-industriesprofessionals/service-industries/veterinary-surgeons/footmouth-info/clinical-signs-cattle, and in human oral cavity (right), available at https://www.medicinenet.com/imagecollection/hand-foot-and-mouth_disease_in_mouth_1_picture/ picture.htm.

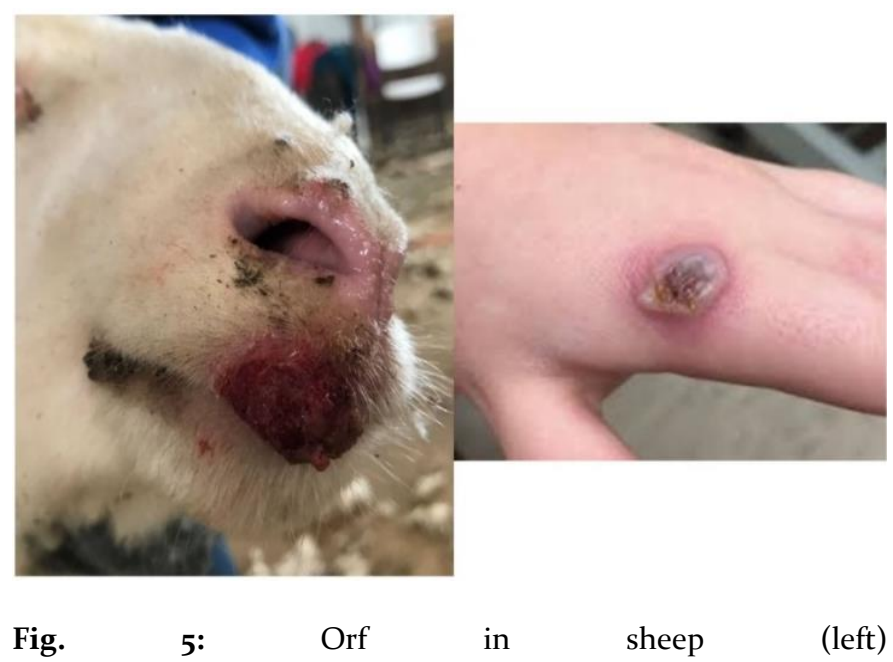

(https://u.osu.edu/sheep/2019/04/23/soremouth-orf-in-smallruminants/), and in human (right) https://dermnetnz.org/ topics/orf/.

\section{Pathogenesis}

The virus enters the body via injured skin and then replicates in the cytoplasm of epithelial cells. At the portal of entry, the primary skin lesion develops 2-6 days post infection, but there is no viremia. The virus replicates whichleads to edema and granulomatous lesions in the dermal layer. Typical lesions are erythema, papules, vesicles and pustules with yellowish creamy exudate, and scabs that become dry and shed with no scar formation. This pattern occurs in 1-2 months (Kumar et al. 2015).

\section{Clinical Signs in Small Ruminants}

In small ruminants, lesions of ORF are seen on the muzzle, lips, nostrils, eyelids, oral mucous membranes, ears and teats of nursing ewes (Fig. 5). Severe pain can interfere with feeding, resulting in weight loss. The lesions of the udder are due to contamination during lactation and cause mastitis. Enlargement of the lymph nodes, pneumonia and arthritis, resulting from the sore mouth have also been recorded. Necrosis of the mucous membranes of the gastrointestinal tract, skin and urogenital tract can also occur (Karki et al. 2019).

\section{Clinical Signs in Humans}

Incubation period of ORF virus is 3 to 7 days. Large painful nodules distributed on hands have been recorded (Fig. 5); uncommon locations, including scalp, nose, buttocks, axilla and genitalia, have also been recorded. The infection is usually recovered with minimum scarring 1-2 months later. The disease in humans is self-limiting except for immunocompromised patients, who can develop large tumor-like lesions, progressive disease, erythema, multiform reaction including rashes on mucous membranes and skin. Signs in humans also include painful vesicles or necrotic areas on skin, pruritis, axillary lymphadenitis and lymphangitis (Taghipour et al. 2015).

\section{Pathologic Lesions}

Necropsy findings include papules, vesicles, pustules and multifocal-coalescing proliferative and necrotic scabs on hairy skin at the muco-cutaneous junction of lips; these lesions extend to the oral papillae, distal prepuce and medial canthus of the eye. In malignant cases, lesions are congested oral mucosa and upper respiratory tract; in few cases there are esophageal, abomasal, and small intestinal lesions. Lesions are generally proliferative and becomeulcer-like with time (Teshale and Alemayehu 2018).

\section{Clinical Diagnosis}

Diagnosis of ORF may depend on histopathology and clinical manifestation but these are less diagnostic tools. The disease should be differentiated from sheep pox, FMD, Bluetongue, facial eczema, ulcerative dermatosis, staphylococcal dermatitis and Dermatophilosis (Kumar et al. 2015).

\section{Laboratory Diagnosis}

PCR and qPCR have been developed to diagnose the infection. Serological tests such as agar gel precipitation test (AGPT), agglutination test, CFT, ELISA and serum neutralization test (SNT) may help. Virus isolation is the golden standard detection method, but it is time consuming (Tedla et al. 2018).

\section{Treatment}

There is no specific treatment for ORF in animals and humans. Systemic and topical application of antibiotic, antiviral and anti-inflammatory drugs have only limited success. Immune-stimulants are usually indicated. Emaciated animals must be given $10 \%$ glucose saline intravenously. The lesion may be washed with 1:100- 
1:10,0oo potassium permanganate lotion with application of 1:10 boric acid. Supportive treatment of young animals by administering glucose solution through esophageal intubation should be performed (Karki et al. 2019).

\section{Prevention and Control}

In animals, the infection can be prevented by quarantine of newcomers. Vaccination with live virus vaccine is important for minimizing the severity of signs in infected herd; vaccines containing a caprine strain are more effective in goats. Disinfection of animal house and incineration of all infected material are also useful. Slaughterhouses should verify that all animals are in a good condition. Among humans, veterinarians and farmers should wear gloves and face masks while dealing with infected animals. Washing of skin injury with soap and water after contact with animals is also necessary (Teshale and Alemayehu 2018).

\section{Vesicular Stomatitis}

Vesicular Stomatitis (VS) is an infectious disease of cattle, horses and swines. It is caused by vesicular stomatitis virus (VSV), a member of the Vesiculovirus of family Rhabdoviridae. It is an enveloped single stranded RNA virus. VSV has two serotypes, VSV-New Jersey (NJ) and VSV-Indiana (IND). There is no cross reactivity between the two serotypes. Vesicular Stomatitis is endemic in northern South America, southern Mexico, Central America and eastern Brazil. Major epidemics of the disease in cattle and horses in the United States occurred in 1889, 1906, 1916, 1926, 1937, 1949, 1963, 1982, and 1995. The disease spread to Europe during the first World war. The disease is of great economic importance, as attack rates in dairy cattle can reach $96 \%$. Vesicular Stomatitis in humans is considered as a severe but non-fatal infection and the disease takes the form of influenza-like illness (McCluskey et al. 2013).

\section{Epidemiology}

\section{Transmission and environmental risk factors}

Cattle can take the VS infection by injection or aerosol route. Intradermal injection of the virus causes skin lesion at inoculation site, intramuscular injection or aerosol route does not cause such lesion. In epidemic areas, human infection occurs by contact with diseased animals. The role of biting insects may also be suggested. Black fly is the most important insect vector in the United States. Disease is common in tropical and subtropical areas at the end of the rainy season or early in dry season. In Colombia, the outbreak in cattle usually reaches the peak in February and August. The virus is susceptible to many disinfectants like $1 \%$ sodium hypochlorite, aldehydes (e.g. formaldehyde), $40-70 \%$ ethanol, phenolic disinfectants, detergents and $1 \%$ cresylic acid. The virus is also susceptible to ultraviolet light, sunlight and heat (RozoLopez et al. 2018).

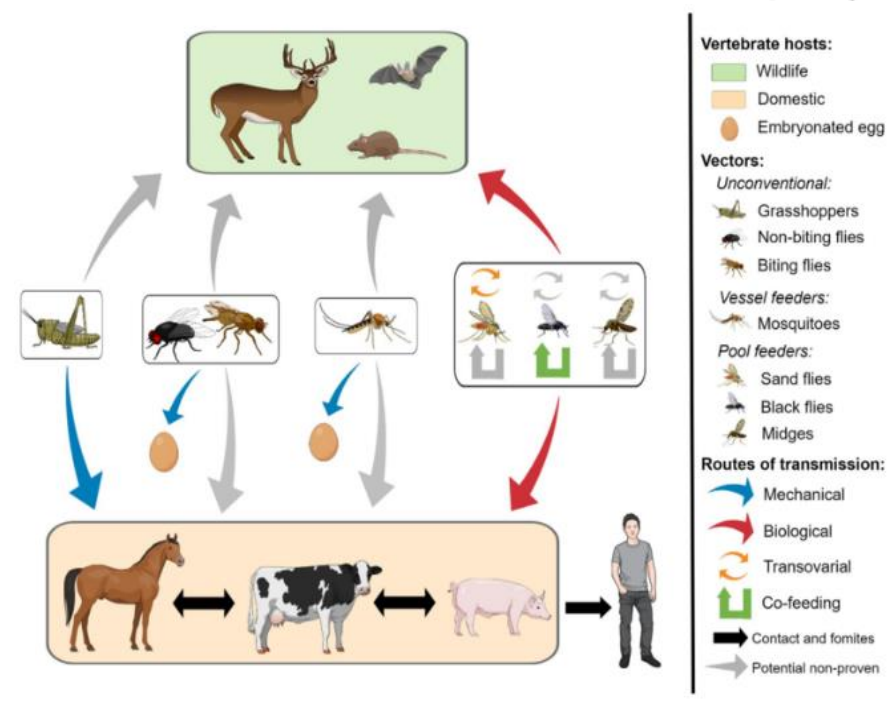

Fig. 6: Transmission networks for vesicular stomatitis (https://www.mdpi.com/2075-4450/9/4/19o/htm)

\section{Geographic distribution}

Vesicular Stomatitis is endemic in Southern Mexico, South America, Venezuela, Colombia, Central America, Ecuador and Peru (Fig. 6). The infection has also been recorded in South Africa in 1886 and 1897 and in France in 1915 and 1917. The annual outbreak of VS occurs in these areas among susceptible species. Outbreaks of VS occurred in all areas of the United States in 1995, 1997, 1998, 2004, 2005, 2006, 2009, 2010, and 2012, but they were limited to western states. The infection spread to Europe during the First World War and appears periodically in South Africa (Reis et al. 2009).

\section{Host range and susceptibility}

Vesicular Stomatitis infection is characterized by a wide host range (cattle, horse and swine). Cattle of less than one year age are asymptomatic. In humans, occupational exposure in laboratory workers and animal handlers, who are exposed to VS, get infected (Smith et al 2012).

\section{Morbidity and mortality}

The morbidity rate in VS is highly variable; it ranges from $5-90 \%$ and is affected by the previous exposure of animal. Only $10-20 \%$ of the animals are usually symptomatic. In non-endemic areas, morbidity rates may reach $40-60 \%$ in susceptible species. Mortalities are very rare in cattle and horses but are higher in pigs. The morbidity in human infection is unknown. Although some authors suggest that clinical cases are rare, other human infections may take the form of influenza-like illness. In most cases, illness has no serious consequences (Rozo-Lopez et al. 2018).

\section{Source of infection}

VS virus sheds from exudates and epithelium of the lesion. Contaminated fomites are suspected to play an 
important role in the virus transmission. The virus survives for about 3-4 days in saliva. Plants and soils are also suspected to be sources of the VS virus infection (Rainwater-Lovett et al 2007).

\section{Pathogenesis}

VSV destroys the cells of prickle cell layer; so large vesicle is formed, which quickly ruptures, leaving erosion or necrotic tissue. The lesion heals quickly (Reis et al. 2009).

\section{Clinical Signs in Cattle}

Incubation period of VS virus infection is 3-7 days. The lesions of VS resemble those of FMD in cattle. Fever occurs in early stages of the disease. Lesions are found on the palate, lips, gums, tongue, coronary band, and teats. Oral lesions are in the form of raised, blanched and fluidfilled vesicles. Vesicles are short-lived and rupture, leaving ulceration or erosions. Lesions may coalesce together to form large, denuded area of oral mucosa with epithelial tags. Lesions are also present on teats of cows and coronary bands of cattle, horses and pigs (Letchworth et al. 1999).

\section{Clinical Signs in Humans}

Incubation period of VS infection in humans is about 1-6 days. Human infection with VSV-NJ and VSV-IND is usually symptomatic. Infection may begin in conjunctiva and is followed by acute, febrile, influenza-like signs as fever, nausea, vomiting, chills, headache, myalgia, malaise, substernal pain, pharyngitis, conjunctivitis, and lymphadenopathy. Encephalitis is a rare sign but may occur in children and may be fatal. Vesicles are also seen in pharynx, tongue or buccal mucosa, or on the skin following direct injection. Signs last 3-6 days and are not associated with complication (Cargnelutti et al. 2014).

\section{Pathologic Lesions}

Lesions at necropsy are the same as seenin live animals. Rumen and heart lesions, which may be found in FMD, do not occur in VS (Letchworth et al. 1999).

\section{Clinical Diagnosis}

Diagnosis of VS may depend on clinical manifestations but they are not usually accurate. The disease should be differentiated from skin affections like sheep pox, FMD and ORF (Cargnelutti et al. 2014).

\section{Laboratory Diagnosis}

The Vesicular Stomatitis virus is isolated from throat swabs, vesicle fluid, saliva and epithelial tissue from the margins of the lesion. Also, electron microscopy, CFT, or FAT can be used. PCR is more sensitive than other methods. Competitive ELISA is used to measure antibodies titer (Letchworth et al. 1999).

\section{Treatment}

This disease has no specific treatment. Anti-inflammatory drugs may help to reduce the swelling and pain. Lesion dressing with mild antiseptic is recommended to prevent secondary bacterial infection. In cases accompanied by fever and inflammation, treatment with antibiotics may be recommended (Cargnelutti et al. 2014).

\section{Prevention and Control}

During outbreak of VS, normal cattle should be kept away from suspected animals. Quarantine and animal movement restrictions can help in reducing the spread of infection. Isolation of symptomatic animals may be also helpful. Disinfection can also minimize the spread of the infection. Feed and water troughs of dairies should be washed regularly. Milking equipment should be sterilized before each use. Avoid thorny food which may predispose the animal to oral lesions. Moving of animals away from sources of running water during outbreak may minimize the risk of infection, as water sources encourage vector populations. Use of insecticides may be helpful. Vaccines are available in some endemic areas of Central and South America. Wearing of protective clothes and gloves while dealing with suspected animals are necessary. Veterinarian who suspects that the animal is infected, must follow his national and/or local guidelines for disease reporting. In the United States, state and federal veterinarians should be reported for any suspected cases immediately (Letchworth et al. 1999).

\section{Pseudocowpox}

Pseudo-cowpox is an infectious disease of cattle worldwide. Infection is caused by member of genus Parapoxvirus, and closely resembles to the viruses of BPS and ORF. Due to the mild signs of the disease in cattle, information about the infection is very little in the literature. The virus has zoonotic importance and farmers are not aware; many times, it remains undiagnosed because of its self-limiting nature. Pseudocowpox virus is one of the two parapoxviruses (PPVs) of cattle, Bovine papular stomatitis virus, previously known as Parapoxvirus bovis-1, and Pseudocowpox virus, previously known as Parapoxvirus bovis-2. Two other virus species are Orf virus of sheep and goats (ORFV, previously known as Parapoxvirus ovis) and Parapoxvirus of red deer in New Zealand (NZPV). Parapoxviruses are epithelia-tropic viruses, identified as causing local vesicles and eruptive skin lesions in wild and domestic animals, especially ruminants (Oguzoglu et al. 2014).

\section{Epidemiology}

\section{Transmission and environmental risk factors}

Transmission of Pseudo-cowpox disease occurs by direct and indirect ways. Indirect route occurs when a calf suckles multiple cows and inadequate milking management procedure. Infection occurs through injured 
skin or occasionally through oral mucosa. The virus infects humans through direct contact. The virus infects the people working with affected cattle with unprotected hands, causing what is called "milker's nodules." (Ouedraogo et al 2020).

\section{Geographic distribution}

The Pseudo-cowpox virus is present worldwide, and affects mainly milking cows but with a limited economic importance. However, in some dairies, this infection may cause some economic losses (Chitala et al. 2020).

\section{Host range and susceptibility}

Recently introduced adult and freshly calved cattle are the most susceptible animal groups to the Pseudocowpox disease. Although, adult cattle including dry cows are affected, cows less than 2 years old appear to be asymptomatic unless they have calved. In humans, the infection is occupational that affects the milkers and other persons in contact with affected cows, the lesion is called Milker's nodule (Craighead 200o).

\section{Morbidity and mortality}

Pseudo-cowpox is often benign with no mortalities, the losses occur due to difficulty in milking and increased incidence of mastitis (Olson et al 2019).

\section{Source of infection}

Fomites, including calves mouths, hands and milking machines are all considered as source of the Pseudocowpox infection (Ouedraogo et al 2020).

\section{Clinical Signs in Cattle}

Lesions of the disease are confined to teats, but may also develop on muzzles and in mouths of nursing calves. Lesions are in the form of ring or horseshoe shaped scabs on teats, and usually heal within 6 weeks. Lesions progress to macules, papules, vesicles, pustules, and scabs (Fig. 7A). Vesicles and pustules covered by scabs can also be seen. Scabs detach after few days in the absence of bacterial contamination, and the lesion heals rapidly (Dhillon et al. 2020).

\section{Clinical Signs in Humans}

Incubation period of Pseudocowpox in humans is 5-7 days. Lesions include small, erythematous papules that appear on hands (Fig. $7 \mathrm{~B}$ and $7 \mathrm{C}$ ); after 4 to 6 weeks they develop into firm nodules of 3-8 $\mathrm{mm}$ diameter before resolution. The disease in humans is similar to 'Orf. No systemic involvement was observed in humans (Dhillon et al. 2020).

\section{Pathologic Lesions}

Localized skin lesions start as a pustule, macule or vesicle that will progressively ulcerate, scab and heal after 5-6 weeks (Samemaleki et al. 2014).

\section{Clinical Diagnosis}

It is important to differentiate the Pseudo-cowpox disease from other diseases causing vesicles, such as FMD and Vesicular Stomatitis (Craighead 200o).

\section{Laboratory Diagnosis}

Samples are taken from scabs of teat lesions. Virus isolation and electron microscopy of lesions are important methods of virus demonstration. Histology and Parapox PCR are also helpful. Clinical and histopathologic characteristics of the disease in humans are indistinguishable and are differentiated based on the history of the initial host exposure (Craighead 200o).

\section{Treatment}

Pseudo-cowpox disease is self-limiting and the lesions should be treated symptomatically through the application of topical ointment to teats and other affected parts. The affected animals should be milked after the healthy ones (Dhillon et al. 2020).

\section{Prevention and Control}

Milking hygiene is an essential measure for the control of Pseudo-cowpox. Affected animals should be milked last. Application of hygienic measures in milking with the use of teat dip may minimize the risk of virus transmission. Acquired immunity is short-lived and there is no vaccination against this virus. Human infections can be reduced by wearing the gloves, thorough hand washing and general precautions when come in contact with infected cows (Craighead 200o).

\section{Rabies}

Rabies is a Latin word for "madness". The Greek name for rabies, lyssa, also means madness. The causative agent of rabies is an RNA virus, belonging to genus lyssavirus and family Rhabdoviridae. This genus has classical rabies virus (RABV, genotype-1) and six rabies-like viruses. Each of them is able to cause rabies-like signs in animals and humans. The virus trans-membrane glycoprotein is important in virus tropism and pathogenicity. It is the main protecting antigen, as it is important in producing the virus neutralizing antibodies. The virus is very sensitive to some environmental factors such as direct sunlight, heat exposure at $60^{\circ} \mathrm{C}$ for 5 minutes and ultraviolet irradiation; it is also sensitive to lipid solvent (70\% alcohol), trypsin, sodium deoxycholate and common detergents (Mtui-Malamsha et al. 2019).

\section{Epidemiology}

\section{Transmission and environmental risk factors}

Rabies virus can infect warm-blooded animals, including humans. Some birds have been known to produce rabies 


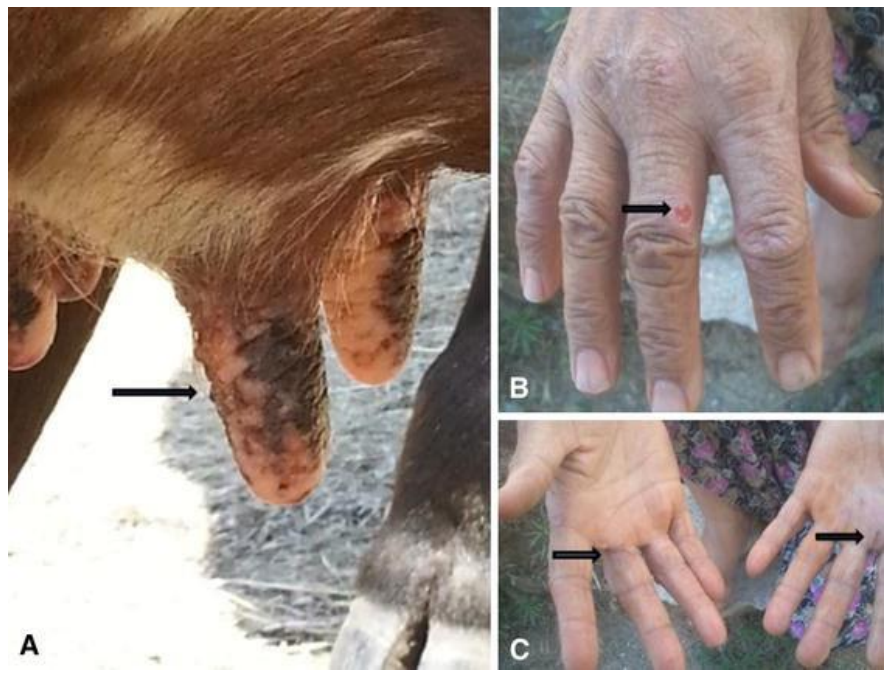

Fig. 7: Pseudocowpox in cattle udder (A) and in human (B and C) (https://link.springer.com/article/10.1007/s13337-014-0214-z).

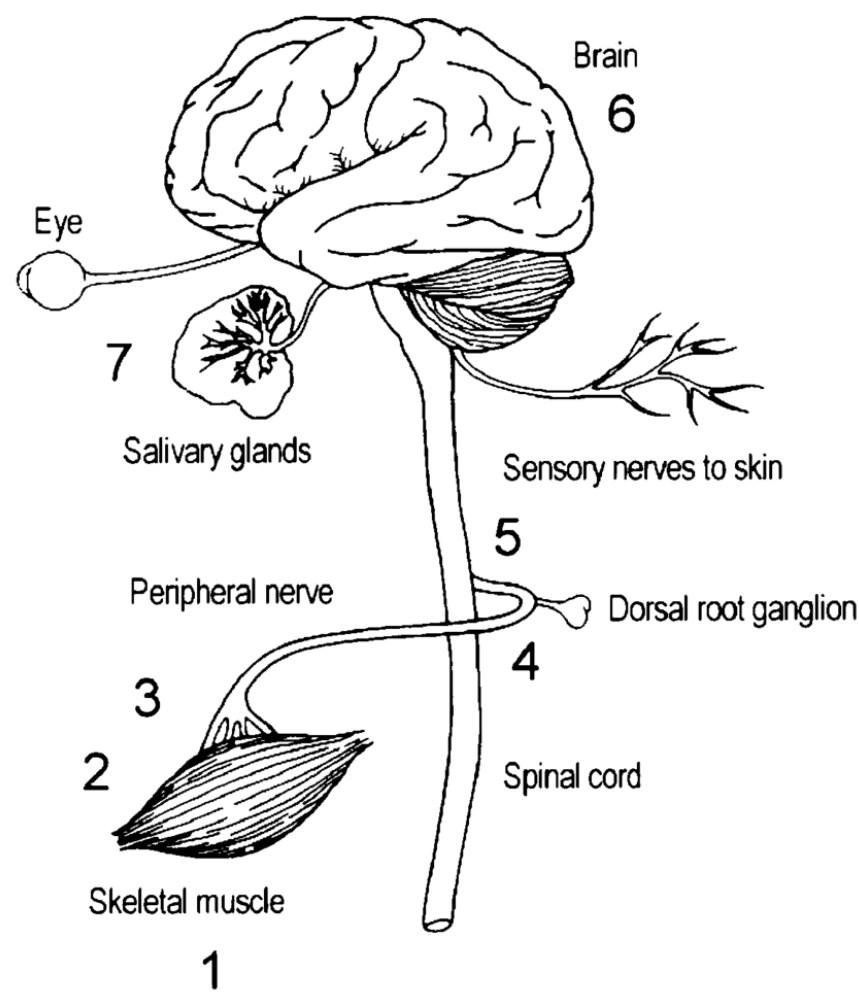

Fig. 8: Pathogenesis of Rabies in man and animals (https://www.researchgate.net/publication/12229458).

antibodies after eating rabid animal. Infected bats, foxes, monkeys, skunks, coyotes, cattle, raccoons, wolves, dogs and cats are considered to be a great risk for humans. Rabies virus can spread via exposure to domestic animals infected with virus. Bites from rodents rarely require rabies prevention, as they are rarely found to be infected. The virus is present in saliva, cerebro-spinal fluid, tears, and nerves of asymptomatic infected animals. The route of infection is mostly through the bite. Transmission from human to human is extremely rare. After infection in humans, the virus is conveyed to peripheral nervous system and is transported along the afferent nerves to central nervous system (Fisher et al. 2018).

\section{Geographic distribution}

Reservoir of rabies virus varies throughout the world. Canine rabies is dominant in Latin America, Africa, Middle East and Asia. In North America and Europe, canine rabies has been totally eliminated; rabies virus is maintained in wildlife. Some countries like United Kingdom, Norway, Iceland, Malaysia, Sweden, New Zealand, Ireland, Singapore, Japan, Australia, Pacific Islands, Papua New Guinea and some Indonesian islands have been recorded as rabies-free areas for many years. About $98 \%$ of human cases are reported from the developing countries of Latin America, Asia and Africa (Singh et al 2017).

\section{Host range and susceptibility}

All warm-blooded animals are susceptible to rabies infection, but limited number of species can act as a reservoir. Cattle with furious form can be dangerous and attack humans and other species. Equines frequently show signs of distress and agitation. Rabid foxes often invade yards and houses and attack dogs and people (Leung et al 2007).

\section{Morbidity and mortality}

Before vaccine development, most human cases of rabies were fatal (Fisher et al. 2018).

\section{Source of infection}

Sources of rabies infection include members of the families Canidae (wolves, dogs, foxes, jackals and coyotes), Viverridae (e.g., mongooses), Mustelidae (e.g., skunks) and Procyonidae (raccoons) and the order Chiroptera (bats). In Africa, primary rabies virus maintenance cycle occurs among domestic dogs, while other carnivores may act as a non-maintenance population (Sugiyamaa and Ito 2007).

\section{Pathogenesis}

Rabies virus enters the body through bite, via injured skin or by contact with mucosa. The virus cannot cross the intact skin. The virus infects local sensory and motor neurons and locally replicates in skeletal muscles or attach directly to the nerve endings, particularly to acetylcholine receptors at motor-end plates. Then the virus migrates in centripetal retrograde axonal transport to CNS (Fig. 8). In the CNS, the virus replicates extensively, and the clinical disease appears. Inflammation is mostly present in the midbrain and medulla in furious stage and the spinal cord in paralytic rabies stage. The virus spreads from the brain to many tissues and organs, replicates in salivary glands and excreted in saliva (Mustafa et al. 2015).

\section{Clinical Signs in Cattle}

Period between the infection and first flu-like symptoms is 2-12 weeks. Incubation period of rabies virus is highly 
variable, based on location and severity of bite and the amount of inoculated virus. Death occurs 2-10 days after the first symptoms. Once symptoms appear, treatment is not effective and the mortality rate is about $99 \%$. Symptoms in animals include change of behavior, loss of appetite, excitement, aggressiveness, paralysis (especially of the lower jaw), fever, change in phonation (sound of a dog's bark) and excessive salivation. All rabid animals exhibit signs of nervous disturbance, with a little variation among species. The clinical course is divided into three phases. 1: Prodromal Phase: This stage usually persists for 1-3 days with minor behavioral changes as aggressiveness, daytime activities and no fear of humans. 2: Excitement (Furious) Phase (mad-dog syndrome): There is rare evidence of paralysis and animal becomes irritable, with dilated pupils, infected animal attacks other animals and people. Muscles incoordination and seizures are common symptoms. As the disease progresses, the death may result from paralysis. 3: Paralytic (Dump) Phase: In this phase, progressive paralysis occurs, throat and masseter muscles get paralyzed and the animals may be unable to swallow. There may be facial paralysis. Ataxia and incoordination are also typical signs of this phase. Death occurs within 26 days due to respiratory failure (Ayele et al. 2018).

\section{Clinical Signs in Humans}

Symptoms of rabies appear within 30-60 days in humans, signs include pain and itching at the site of inoculation, also fever, nausea, restlessness, headache, sore throat and loss of appetite may occur. Excessive salivation, muscle stiffness, excitement or convulsions, increased sensitivity to light or loud sounds are also seen (Mustafa et al. 2015).

\section{Pathologic Lesions}

There is no pathognomonic lesions for rabies, although there may be fresh or healed bite wounds. There is unusual odor related to reduced hygiene. In CNS, there is congestion of the meningeal vessels, the brain tissue may be congested with mild cerebral edema (Ayele et al. 2018).

\section{Clinical Diagnosis}

Clinical diagnosis in this disease is difficult, as Rabies is confused with other diseases with aggressiveness in its early stages. Differential diagnosis includes any infection that causes encephalitis, such as arboviruses, herpesviruses, enteroviruses, tetanus, listeriosis and poisoning (Fisher et al. 2018).

\section{Laboratory Diagnosis}

Frequently used diagnostic method is rabies is FRT (fluorescent rabies test) or fluorescent antibody test-FAT. Microscopic examination of tissue helps in identification of virus-specific antigen. RT PCR is the sensitive and specific test (Fisher et al. 2018).

\section{Treatment}

After viral exposure, treatment is able to prevent the infection if it is taken within 10 days post-infection. Thorough washing of skin with soap and water is effective; use of povidone-iodine and alcohol is effective in reducing the risk of development of the disease. It is necessary to take one dose of human rabies immunoglobulin and also 4 doses of rabies vaccine over 14 days period. Post exposure prophylaxis should be given without delay (Mustafa et al. 2015).

\section{Prevention and Control}

Vaccination before exposure has been done in human and non-human species, with the domestic animals are important to be vaccinated. Control measures should be appliedlike, vaccination of dogs, ferrets and cats, keeping pet animals under good supervision, avoid handling wild and stray animals, contacting the veterinarian when observing wild or stray animals and also washing the site of exposure with soap and water for 10-15 min post exposure (Mtui-Malamsha et al. 2019).

\section{REFERENCES}

Al-Salihi KA, 2018. Camelids zoonotic diseases. Journal of Camelid Science 11: 1-20.

Al-Tayib OA, 2019. An overview of the most significant zoonotic viral pathogens transmitted from animal to human in Saudi Arabia. Pathogens 8(25): 1-32.

Arzt J et al., 2011. The pathogenesis of Foot-and-Mouth Disease I: Viral pathways in cattle. Transboundary Emergency Diseasese 58(4): 291-304.

Aslam S, 2016. Crimean-Congo hemorrhagic fever: Risk factors and control measures for the infection abatement (review). Biomedical Reports. 4: 15-20.

Ayele $\mathrm{T}$ et al., 2018. Review on Rabies and its zoonotic importance. Academic Journal of Animal Diseases $7(2): 29-38$.

Bauer K, 1997. Foot-and-Mouth Disease as a zoonosis. Annual Review of Microbiology 22: 201-244.

Boshra $\mathrm{H}$ et al., 2011. Rift Valley Fever: Recent insights into pathogenesis and prevention. Journal of Virology 85(13): 6098-6105.

Cargnelutti JF et al., 2014. Outbreaks of vesicular stomatitis Alagoas virus in horses and cattle in northeastern Brazil. Journal of Veterinary Diagnostic Investigations 26(6): 788-794.

Chitala $C$ et al., 2020. First detection and molecular characterisation of Pseudocowpox virus in a cattle herd in Zambia Maureen. Virology Journal 17(1): 152.

Craighead JE, 20oo. In: Pathology and Pathogenesis of Human Viral Disease. Elsevier Ltd.

deSant'Ana JF et al, 2012. Bovine papular stomatitis affecting dairy cows and milkers in midwestern Brazil. Journal of Veterinary Diagnostic Investigations 24(2): 442-445.

Dhillon KS et al., 2020. Clinical management of Pseudocowpox and its zoonotic significance: A report of 
three cows. Journal of Entomology and Zoology Studies 8(2): 1939-1941.

Elmeligy EE, 2017. Foot and Mouth Disease. SOJ Veterinary Sciences 3(4): 1-2.

Ermias D et al., 2017. Zoonotic disease programs for enhancing global health security. Emerging Infectious Diseases 23: $665-\mathrm{S} 70$.

Farsang A et al., 2013. Control of the deliberate spread of Foot-and-Mouth Disease virus. Biosecurity and Bioterrorism: Biodefense Strategy, Practice, and Science 11(Supplement 1): 115-122.

Fisher CR et al., 2018. The spread and evolution of Rabies virus: conquering new frontiers. Nature Reviews Microbiology 16(4): 241-255.

Ganter M, 2015. Zoonotic risks from small ruminants. Veterinary Microbiology 181(1-2): 53-65.

Garvey M, 2018. Zoonotic parasite species and viral pathogens of livestock associated with human morbidity. EC Veterinary Science 3(2): 300-311.

Gelberg HB, 2017. In: Pathologic Basis of Veterinary Disease. 6th Edition, Elsevier Ltd.

Helmy YA et al., 2017. A comprehensive review of common bacterial, parasitic and viral zoonoses at the human-animal interface in Egypt. Pathogens 6: 1-28.

Javelle et al., 2020. The challenging management of Rift Valley Fever in humans: literature review of the clinical disease and algorithm proposal. Annals of Clinical Microbiolology and Antimicrobials 19: 4 https://doi.org/10.1186/s12941-020-0346.

Joseph RH et al., 2015. Erythema multiforme after orf virus infection: a report of two cases and literature review. Epidemiology and Infection 143(2): 385-390.

Karki $\mathrm{M}$ et al., 2019. Contagious ecthyma of sheep and goats: A comprehensive review on epidemiology, immunity, diagnostics and control measures. Veterinarski Archive 89(3): 393-423.

Kasye $\mathrm{M}$ et al., 2016. A review on Rift Valley Fever on animal, human health and its impact on livestock marketing. Austin Virology and Retrovirology 3(1): 1020.

Kumar R et al., 2015. Contagious pustular dermatitis (orf disease) - epidemiology, diagnosis, control and public health concerns. Advances in Animal and Veterinary Sciences 3(12): 649-676.

Letchworth GJ et al., 1999. Vesicular stomatitis. The Veterinary Journal 157: 239-260.

Leung AKC et al., 2007. Rabies: Epidemiology, pathogenesis, and prophylaxis. Adv Therapy 24: 13401347.

Lichoti JK, 2014. Detection of Rift Valley Fever virus interepidemic activity in some hotspot areas of Kenya by Sentinel Animal Surveillance, 2009-2012. Veterinary Medicine International 2014: 379010. http://dx.doi.org/10.1155/2014/379010.

Martinez-Salas E et al., 20o8. "Foot-and-Mouth Disease Virus". Animal Viruses: Molecular Biology. Caister Academic Press. pp. 1-38.

McCluskey BJ et al., 2013. Vesicular stomatitis outbreak in the southwestern United States. Journal of Veterinary Diagnostic Investigations 25(5): 608-613.
McDanie CJ et al., 2014. Humans and cattle: A review of bovine zoonoses. Vector-Borne and Zoonotic Diseases 14(1): DOI: 10.1089/vbz.2012.1164.

Métrasa $\mathrm{R}$ et al., 2020. Estimation of Rift Valley Fever virus spillover to humans during the Mayotte 20182019 epidemic. Proceedings of the National Academy of Sciences, USA 117(39): 24567-24574.

Mostafavia E et al., 2017. Seroepidemiology and risk factors of Crimean-Congo Hemorrhagic Fever among butchers and slaughterhouse workers in southeastern Iran. International Journal of Infectious Diseases 64: $85-89$.

Mtui-Malamsha $\mathrm{N}$ et al., 2019. Ecological and epidemiological findings associated with zoonotic Rabies outbreaks and control in Moshi, Tanzania, 2017-2018. International Journal of Environmental Research and Public Health 16: 1-14.

Munibullah et al, 2018. Crimean-Congo hemorrhagic fever: a threat to public health. Journal of Bacteriology and Infectious Diseases 2(1): 1-7.

Mustafa $\mathrm{M}$ et al., 2015. Rabies a zoonotic disease, transmission, prevention, and treatment. IOSR Journal of Dental and Medical Sciences 14(10): 79-84.

Nakoune E et al., 2016. Rift Valley Fever virus circulating among ruminants, mosquitoes and humans in the central African Republic. PLoS Neglected Tropical Diseases 10(10): e0005082.

Oguzoglu TC et al., 2014. Evidence of zoonotic Pseudocowpox virus infection from a cattle in Turkey. Virus Disease 25(3): 381-384.

Olson VA, et al., 2019. Poxviruses. Manual of Clinical Microbiology, $12^{\text {th }}$ Edition. ASM Press, Washington DC, USA.

Osmani IDR et al., 2019. History and epidemiology of Foot-and Mouth Disease in Afghanistan: a retrospective study. BMC Veterinary Research 15:340.

Ouedraogo A et al., 2020. Detection of two species of the genus Parapoxvirus (Bovine Papular Stomatitis virus and Pseudocowpox virus) in ticks infesting cattle in Burkina Faso. Microorganisms 8(5): 644; doi: 10.3390/microorganisms8050644 1-10.

Pal M, 2020. Is Bovine Papular Stomatitis an infectious viral zoonosis? CPQ Microbiology 3(4): 01-03.

Parmar BC et al, 2017. Crimean-Congo Haemorrhagic Fever: A fatal viral zoonotic disease. Trends in Veterinary and Animal Sciences 4: 4-6.

Peek SF et al., 2018. In: Rebhun's Diseases of Dairy Cattle. $3^{\text {rd }}$ Edition. Elsevier Ltd.

Petrova V et al., 2020. Rift valley fever: diagnostic challenges and investment needs for vaccine development. British Medical Journal Global Health 5(8): e002694. doi: 10.1136/ bmjgh-2020-002694.

Rainwater-Lovett K et al., 2007. Molecular epidemiology of vesicular stomatitis New Jersey virus from the 2004-2005 US outbreak indicates a common origin with Mexican strains. Journal of General Virology 88: 2042-2051.

Reed KD, 2018. Viral Zoonoses. Reference Module in Biomedical Sciences. https://doi.org/10.1016/B978-o12-801238-3.95729-5 1-12 Elsevier. 
Reis jr et al., 2009. Transmission and pathogenesis of vesicular stomatitis viruses. Brazilian Journal of Veterinary Pathology 2(1): 49-58.

Rozo-Lopez P et al., 2018. Vesicular Stomatitis Virus transmission: A comparison of incriminated vectors. Insects 9: 190; doi: 10.3390/insects9040190.

Rweyemamu M et al., 2008. Epidemiological patterns of Foot-and-Mouth disease worldwide. Transboundary and Emerging Diseases 55: 57-72.

Samemaleki E et al., 2014. Case report of pseudocowpox in a 3 years old cow and the resulting lesions in a 35 years old woman. European Journal of Experimental Biology 4(1): 142-144.

Senturk S et al., 2016. Outbreak of Bovine Papular Stomatitis with concurrent Cryptosporidiosis in a dairy herd in Turkey. Bulgarian Journal of Veterinary Medicine 1: 78-83.

Shayan S et al., 2015. Crimean-Congo Hemorrhagic Fever. Laboratory Medicine 46(3): 180-189.

Shayif AA et al., 2018. Epidemiological study on Rift Valley Fever virus among humans in Taiz Governorate (Yemen). American Journal of Clinical Microbiology and Antimicrobials 1(6): 1028.

Singh R et al., 2017. Rabies - epidemiology, pathogenesis, public health concerns and advances in diagnosis and control: a comprehensive review. Veterinary Quarterly 37(1): 212-251.

Smith PF et al., 2012. Host predilection and transmissibility of vesicular stomatitis New Jersey virus strains in domestic cattle (Bos taurus) and swine (Sus scrofa). BMC Veterinary Research 8: 183.

Sugiyamaa $M$ and Ito $N$, 2007. Control of rabies: Epidemiology of rabies in Asia and development of new-generation vaccines for rabies. Comparative Immunology, Microbiology and Infectious Diseases 30: $273-286$.

Taghipour M, 2015. Orf virus infection in human (echtyma contagiosum): A report of eight cases in the north of Iran. Medical Investigations 4(1): 183-186.

Tedla $\mathrm{M}$ et al., 2018. Molecular identification and investigations of contagious ecthyma (Orf virus) in small ruminants, North west Ethiopia. BMC Veterinary Research 14: 13. doi: 10.1186/s12917-018-1339-X.

Teshale A and Alemayehu A, 2018. Contagious ecthyma and its public health significance. Dairy and Veterinary Sciences Journal 7(3): 1-6.

Underwood WJ et al., 2015. In: Laboratory Animal Medicine. $3^{\text {rd }}$ Ed. Elsevier Ltd.

Walter E and MikeScott M, 2017. The life and work of Rudolf Virchow 1821-1902: Cell theory, thrombosis and the sausage duel. Journal of Intensive Care Society 18(3): 234-235.

Weaver JV et al., 2013. Foot and Mouth Disease: A look from the wild side. Journal of Wildlife Diseases, 49(4): 759-785.

Zavitsanou A et al., 2009. Crimean Congo hemorrhagic fever: an emerging tick-borne disease. Health Science Journal 3(1): 10-18. 